\title{
Burnout, workaholism e qualidade de vida entre docentes de pós-graduação em enfermagem
}

Burnout, workaholism and quality of life among professors in graduate-level nursing programs Burnout, workaholism y calidad de vida de docentes de posgrado en enfermería

Maria José Quina Galdino ${ }^{1}$ id https://orcid.org/0000-0001-6709-3502

Julia Trevisan Martins² io https://orcid.org/0000-0001-6383-7981

Maria Lucia do Carmo Cruz Robazzi ${ }^{3}$ io https://orcid.org/0000-0003-2364-5787

Sandra Marisa Pellos0 ${ }^{4}$ io https://orcid.org/0000-0001-8455-6839

Maynara Fernanda Carvalho Barreto ${ }^{1}$ io https://orcid.org/0000-0002-3562-8477

Maria do Carmo Fernandez Lourenço Haddad² io https://orcid.org/0000-0001-7564-8563

Gomo citar: SM, Barreto MF, Haddad MC. Burnout, workaholism e qualidade de vida entre docentes de pós-graduação em enfermagem. Acta Paul Enferm. 2021;34:APE00451.

DOI http://dx.doi.org/10.37689/actaape/2021A000451

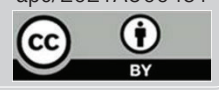

Descritores

Esgotamento psicológico; Qualidade de vida; Educação de pós-graduação em enfermagem; Docentes de enfermagem; Condições de trabalho

Keywords

Burnout, psychological; Quality of life; Education, nursing, graduate; Faculty, nursing; Working

conditions

Descriptores

Agotamiento psicológico; Calidad de vida; Educación de postgrado en enfermería; Docentes de enfermería; Condiciones de trabajo

Submetido 8 de Março de 2020 Aceito 22 de Abril de 2020

Autor correspondente Maria José Quina Galdino E-mail: mjqgaldino@gmail.com

\section{Resumo}

Objetivo: Verificar a associação do burnout com workaholism e qualidade de vida entre docentes de mestrado e/ou doutorado em enfermagem.

Métodos: Estudo transversal realizado com docentes permanentes vinculados aos Programas de PósGraduação da área da Enfermagem de 47 universidades públicas das cinco regiões do Brasil. Entre julho e dezembro de 2018, convidou-se 919 docentes, dos quais 368 responderam a quatro questionários: caracterização sociodemográfica, de saúde e ocupacional, Maslach Burnout Inventory - Human Services Survey, Dutch Work Addiction Scale e World Health Organization Quality of Life Assessment Instrument Bref. Os dados foram analisados por estatística descritiva e as associações foram verificadas por regressão logística múltipla bruta e ajustada.

Resultados: A prevalência de indicativo de burnout foi de $28,0 \%$, de workaholism foi de $35,5 \%$ e de baixa qualidade de vida geral foi de $17,7 \%$ entre os docentes permanentes investigados. Trabalho compulsivo, trabalho excessivo e ser workaholic aumentaram significativamente as chances de altos níveis de exaustão emocional, despersonalização e baixa eficácia profissional. Por outro lado, níveis significativamente menores foram observados nos trabalhadores positivos, com alta percepção de qualidade de vida geral, física, psicológica, social e do meio ambiente. Os modelos múltiplos das dimensões da síndrome indicaram que suas dimensões são direta e positivamente associadas com 0 workaholism e direta e negativamente associadas à qualidade de vida geral, mesmo após o ajuste com variáveis sociodemográficas, de saúde e ocupacionais.

Conclusão: A síndrome de burnout foi associada aos professores de mestrado e/ou doutorado com workaholism e que consideravam ter uma baixa qualidade de vida.

\section{Abstract}

Objective: To verify the association of burnout between workaholism and quality of life among graduate-level nursing professors.

Methods: A cross-sectional study was conducted with permanent professors linked to Postgraduate Programs in Nursing at 47 public universities in the five regions of Brazil. Between July and December 2018, 919 teachers were invited, of whom 368 answered four questionnaires: sociodemographic, health and occupational characterization, Maslach Burnout Inventory - Human Services Survey, Dutch Work Addiction Scale and World Health Organization Quality of Life Assessment Instrument - Bref. The data were analyzed using descriptive statistics and the associations were verified by unadjusted and adjusted multiple logistic regression.

'Universidade Estadual do Norte do Paraná. Bandeirantes, PR, Brasil.

2Universidade Estadual de Londrina. Londrina, PR, Brasil

${ }^{3}$ Escola de Enfermagem de Ribeirão Preto. Universidade de São Paulo. Ribeirão Preto, SP, Brasil.

${ }^{4}$ Universidade Estadual de Maringá. Maringá, PR, Brasil.

Conflitos de interesse: nada a declarar. 
Results: The prevalence of indicative of burnout was $28.0 \%$, of workaholism was $35.5 \%$ and of low general quality of life was $17.7 \%$ among the permanent professors investigated. Working excessively, working compulsively and being a workaholic significantly increased the chances of high levels of emotional exhaustion, depersonalization and low professional accomplishment. On the other hand, significantly lower levels were observed in the positive professionals, with a high perception of general, physical, psychological, social, and environmental quality of life. The multiple models of the syndrome indicated that its dimensions are directly and positively associated with workaholism and directly and negatively associated with the general quality of life, even after adjustment with sociodemographic, health and occupational variables.

Conclusion: Burnout was associated with professors with workaholism and those who consider themselves as having a poor quality of life.

\section{Resumen}

Objetivo: Verificar la relación del burnout con el workaholism y la calidad de vida de docentes de maestría y doctorado en enfermería.

Métodos: Estudio transversal realizado con docentes permanentes vinculados a los Programas de Posgrado del área de Enfermería de 47 universidades públicas de las cinco regiones de Brasil. Entre julio y diciembre de 2018, se invitó a 919 docentes, de los cuales 368 respondieron cuatro cuestionarios: caracterización sociodemográfica, de salud y ocupacional, Maslach Burnout Inventory - Human Services Survey, Dutch Work Addiction Scale y World Health Organization Quality of Life Assessment Instrument - Bref. Los datos fueron analizados mediante estadística descriptiva y las relaciones fueron verificadas mediante regresión logística múltiple bruta y ajustada.

Resultados: La prevalencia de indicios de burnout fue del 28,0 \%, de workaholism del 35,5\% y de baja calidad de vida general del $17,7 \%$ entre los docentes permanentes investigados. Trabajo compulsivo, trabajo en exceso y ser workaholic aumentaron significativamente la probabilidad de altos niveles de agotamiento emocional, despersonalización y baja eficacia profesional. Por otro lado, se observaron niveles significativamente menores en trabajadores positivos, con una alta percepción de calidad de vida general, física, psicológica, social y del medio ambiente. Los modelos múltiples de las dimensiones del síndrome indicaron que sus dimensiones están directa y positivamente relacionadas con el workaholism y directa y negativamente relacionadas con la calidad de vida general, inclusive después de ajustar las variables sociodemográficas, de salud y ocupacionales.

Conclusión: El síndrome de burnout está relacionado con profesores de maestría y doctorado con workaholism y que consideran que tienen una mala calidad de vida.

\section{Introdução}

As atividades dos professores exigem muitas horas diárias de trabalho e intenso esforço mental. ${ }^{(1)}$ Isso os predispóe a trabalhar por um período excessivo de tempo e colabora na criação de uma compulsão pelo labor, na tentativa de obter sucesso constante, levando ao vício em trabalho. ${ }^{(2)}$

O workaholism refere-se à dependência psicológica e patológica do indivíduo ao seu trabalho, que caracteriza-se por trabalhar de forma excessiva e compulsiva para atender aos padróes organizacionais, negligenciando o descanso e a vida pessoal. Assim, têm-se as duas dimensóes do workaholism: trabalho excessivo, a dimensão comportamental, e trabalho compulsivo, a dimensão cognitiva. ${ }^{(3)}$

O trabalho em excesso interfere na relação de satisfação e prazer que o indivíduo tem com o labor e na qualidade de vida, visto que a sua percepçáo é temporal e circunstancial. ${ }^{(4)}$ A qualidade de vida relaciona-se à percepçáo subjetiva do indivíduo sobre o bem-estar no seu contexto de vida, em uma perspectiva que inter-relaciona condição física, psicológica, relaçóes sociais e aspectos referentes ao ambiente onde vive. ${ }^{(5)}$
Estudos indicam que o workaholism e os altos níveis de exigências frente às complexas atividades desempenhadas pelo professor possuem relação significante e positiva com o burnout. ${ }^{(2,6)}$ As investigaçóes com enfermeiros avaliaram que a baixa qualidade de vida, ${ }^{(7)}$ e o workaholism $^{(8,9)}$ foram relacionados ao burnout.

Burnout é uma síndrome psicológica em resposta à sobrecarga e aos estressores interpessoais crônicos provenientes do ambiente ocupacional e resulta no esgotamento do indivíduo. As três dimensôes desta resposta são um cansaço acentuado, sentimentos de cinismo, desapego laboral e ineficácia profissional. ${ }^{(9,10)}$

$\mathrm{O}$ desgaste do professor tem consequências devastadoras para ele e para a qualidade da educação, como menor satisfação laboral, menores níveis de comprometimento, intenção de abandonar sua posição docente e absenteísmo. ${ }^{(1,11,12)}$ A síndrome impacta a saúde com problemas cardiovasculares, respiratórios, gastrointestinais, musculoesqueléticos, endócrinos, alterações nas experiências álgicas, ferimentos graves e mortalidade abaixo dos 45 anos, distúrbios de sono e depressão, além de outras repercussóes organizacionais, como presenteísmo e aposentadoria por invalidez. ${ }^{(13)}$ 
Workaholism e qualidade de vida podem estar associados ao burnout. No entanto, são incipientes as pesquisas que identificaram a relação desses fenômenos com o burnout, sobretudo entre docentes de mestrado e/ou doutorado, pois há pouco conhecimento produzido sobre suas condiçôes de trabalho, não apenas como uma atividade em si, mas como uma atividade entre outras atividades acadêmicas nas universidades. ${ }^{(14)}$ Compreender essa relação possibilitará a elaboração de intervençôes para favorecer a qualidade de vida e a saúde desses indivíduos, produzindo um ambiente favorável ao aprendizado e à formação acadêmica de pesquisadores.

Hipotetiza-se que as dimensóes de burnout são direta e positivamente associadas ao workaholism e direta e negativamente associadas a qualidade de vida, independente de sexo, idade, depressão, regime de trabalho, receber bolsa produtividade, anos de docência no mestrado/doutorado, número de Programas de Pós-graduação (PPG) de vínculo e orientandos mestrado/doutorado, conforme apresentado na figura 1.

Isto posto, este estudo objetivou verificar a associação do burnout com workaholism e qualidade de vida entre docentes de mestrado e/ou doutorado em enfermagem.

\section{Métodos}

Pesquisa quantitativa e transversal realizada entre julho e dezembro de 2018 com docentes de 51 PPG da área de Enfermagem de 47 universidades públicas brasileiras.

A população convidada para participar do estudo compreendeu 919 docentes (dados extraídos da Plataforma Sucupira em maio de 2018), os quais atendiam aos seguintes critérios de elegibilidade: (1) ser professor permanente, designação atribuída àqueles que desenvolvem atividades de ensino na pós-graduação e graduação, participam de projetos de pesquisa do PPG, orientam alunos de mestrado ou doutorado no PPG e têm vínculo institucional; (2) estar credenciado há, no mínimo, um ano a pelo menos um dos PPG em estudo; e (3) não estar afastado por licenças de qualquer natureza.

O tamanho amostral foi calculado utilizando a fórmula $n=N \cdot p \cdot q \cdot(Z \alpha / 2)^{2} /$ p.q. $(Z \alpha / 2)^{2}+(N-1)$. $(E)^{2}$, em que adotou-se proporção de $50 \%$, intervalo de confiança de $95 \%$ e erro máximo de $5 \%$, resultando no mínimo de 270 participantes. Considerando eventuais perdas, convidamos os 919 docentes elegíveis, dos quais 368 (40,1\%) responderam à essa pesquisa.

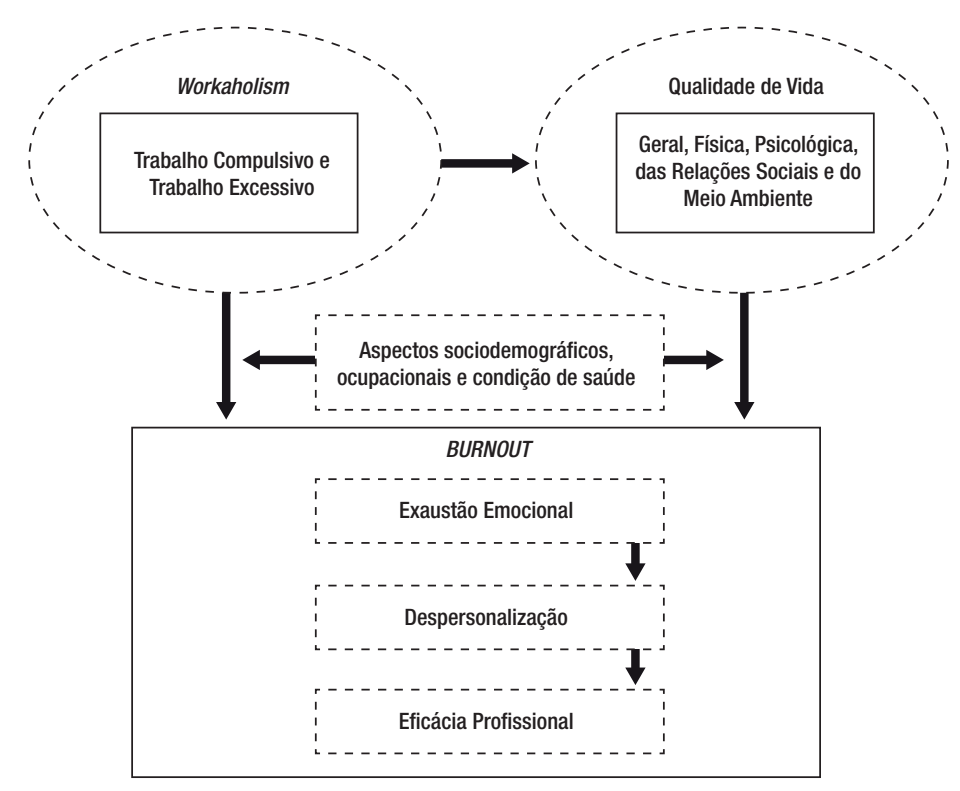

Figura 1. Modelo hipotético da associação do burnout com workaholism e qualidade de vida 
Os dados de caracterização sociodemográfica, saúde e trabalho foram coletados por um questionário com as informaçóes: idade, sexo, filhos, situação conjugal, uso de antidepressivos, área de graduação, regime de trabalho, receber bolsa produtividade, tempo de docência no mestrado/doutorado, número de PPG de vínculo e número de orientados no mestrado/doutorado.

A versão brasileira do Maslach Burnout Inventory -Human Services Survey (MBI-HSS) foi usada para verificar o burnout, pois possui os requisitos necessários quanto a validade fatorial e consistência interna. ${ }^{(15)}$ Trata-se de um instrumento de 22 itens com respostas tipo Likert de 7 pontos (0: nunca a 6: diariamente), que dividem-se em três dimensôes: exaustão emocional, despersonalização e eficácia profissional.

Verificou-se o workaholism pela Dutch Work Addiction Scale (DUWAS) na versão português do Brasil, que possui validade fatorial e confiabilidade adequadas. ${ }^{(16)} \mathrm{O}$ instrumento é composto de 10 itens com respostas tipo Likert de 4 pontos (1: nunca a 4: sempre), que geram duas dimensóes: trabalho compulsivo e excessivo. A combinação das dimensôes da DUWAS emerge dois perfis: trabalhador positivo (baixa pontuação em trabalho excessivo e compulsivo) e workaholics (alta pontuação em trabalho excessivo e compulsivo). ${ }^{(3)}$

Avaliou-se a qualidade de vida pela versão brasileira do World Health Organization Quality of Life Assessment Instrument - Bref (WHOQOL-Bref), que contém 26 itens, sendo dois gerais e os demais agrupados em quatro domínios: qualidade de vida física, psicológica, das relaçóes sociais e do meio ambiente. As respostas têm formato de Likert de cinco pontos, que geram um escore por domínios e, por meio da fórmula $((\bar{x}$ domínio*4)-4 $\left.)^{*}(100 / 16)\right)$, são transformados em uma escala linear que varia de 0: qualidade de vida menos favorável a 100: qualidade de vida mais favorável. ${ }^{(5)}$

Esses instrumentos foram inseridos em uma plataforma na internet, desenvolvida pela equipe de pesquisa para a coleta dos dados. Configurou-se o sistema para os itens fossem de preenchimento obrigatório, evitando-se dados missing, bem como para que o envio dos convites ocorresse cada 15 dias, sendo automaticamente excluídos da lista os docentes que não respondessem até a quinta tentativa. Os convites foram enviados por e-mail, cujo conteúdo constou de esclarecimentos sobre a pesquisa e um botão de acesso à plataforma on-line.

$\mathrm{O}$ sistema gerou uma planilha com as respostas dos participantes que foi codificada para ser analisada no Statistical Package of Social Sciences (SPSS), versão 20.0. Estatísticas descritivas foram usadas para descrever todas as variáveis de estudo. As variáveis numéricas não aderiam a distribuição normal (ShapiroWilk p<0,001), assim, houve descrição por medianas e intervalos interquartílicos (IIQ) (p25-p75).

As variáveis dependentes do estudo foram as dimensões do burnout: exaustão emocional, despersonalização e eficácia profissional. As variáveis independentes foram as dimensôes do workaholism: trabalho excessivo e compulsivo, e seus perfis: workaholic e trabalhador positivo; e os domínios da qualidade de vida: overall, físico, psicológico, relaçôes sociais e meio ambiente. Todas as pontuaçôes dessas dimensôes/domínios foram dicotomizadas em alto e baixo, considerando a mediana como ponto de corte.

Realizaram-se regressóes logísticas binárias univariadas para verificar a associação isolada das variáveis dependentes com as independentes. As regressóes foram repetidas em modelos múltiplos, inserindo-se as variáveis de ajuste: sexo, idade, anos de docência no mestrado/doutorado e depressão pela literatura indicar como aspectos a serem controlados. $^{(11)}$ e regime de trabalho, número de PPG de vínculo, receber bolsa produtividade e número de orientados no mestrado/doutorado, por considerarmos prováveis confundidores da relação. Por fim, foram elaborados modelos múltiplos inserindo a variável workaholism (perfil workaholic) e a qualidade de vida geral (domínio overall), que também foram controlados pelas variáveis de ajuste supracitadas. Verificou-se a qualidade do ajuste do modelo e a variação explicada do modelo múltiplo sobre o desfecho pelos testes de Hosmer-Lemeshow e Nagelkerke R Square, respectivamente. Os resultados foram descritos por odds ratio (OR) brutos e ajustados com intervalos de confiança de 95\%.

$\mathrm{O}$ estudo foi desenvolvido atendendo aos princípios de ética em pesquisa envolvendo seres humanos, sendo aprovado por Comitê de Ética em Pesquisa, conforme Parecer n. ${ }^{o}$ 2.347.839. 


\section{Resultados}

Participaram deste estudo 368 docentes com idade mediana de 53 anos (IIQ: 17), sendo a mínima de 28 e máxima de 75 anos. A maioria era mulher $(84,5 \%)$, possuía filhos $(73,9 \%)$ e relacionamento conjugal estável (69,6\%). Predominaram docentes graduados em enfermagem $(90,5 \%)$, seguidos de outras áreas da saúde $(5,7 \%)$ e de outras áreas do conhecimento (3,8\%). Quanto ao regime de trabalho, 91,3\% exerciam dedicação exclusiva ou integral. Verificou-se que $19 \%$ recebiam bolsas de produtividade em pesquisa. $\mathrm{O}$ tempo de docência no mestrado e/ou doutorado foi, em mediana, 8 anos, com variaçóes de 1 a 40 anos. A maioria estava credenciada à um PPG (68,5\%), mas 27,7\% e $3,8 \%$ estavam vinculados a dois ou três, respectivamente. $\mathrm{O}$ número de orientandos no mestrado e/ ou doutorado variou entre 1 e 17, com mediana de 5 pós-graduandos. Em relação a condição de saúde, $13,6 \%$ indicaram uso de antidepressivos.

A alta exaustão emocional ocorreu com $49,7 \%$ dos docentes, a alta despersonalização com 47,0\% e a ineficácia profissional com $51,1 \%$, que ao serem combinados indicaram uma prevalência de $28,0 \%$ de burnout. Constatou-se que 49,7\% apresentaram alto trabalho compulsivo e $45,7 \%$ alto trabalho excessivo e a junçáo dessas dimensôes indicou que $35,1 \%$ apresentavam workaholism e 39,7\%, trabalho positivo. Sobre a qualidade de vida, a percepção alta do domínio overall ocorreu com $17,7 \%$; do físico, 55,7\%; do psicológico, 60,1\%; das relaçóes sociais, com 49,2\%; e do meio ambiente onde está inserido, $43,5 \%$.

Alto trabalho compulsivo, alto trabalho excessivo e ser workaholic aumentaram significativamente as chances de alta exaustáo emocional, alta despersonalização e baixa eficácia profissional. Todavia, as chances foram diminuídas significativamente para trabalhador positivo, alta pontuação na qualidade de vida geral, física, psicológica, social e do meio ambiente (Tabela 1).

$\mathrm{Na}$ tabela 2 estáo apresentados os modelos múltiplos da associação das dimensóes da síndrome de burnout com o Workaholism e do domínio overall da qualidade de vida.
Tabela 1. Associações entre burnout, workaholism e qualidade de vida na amostra em estudo

\begin{tabular}{|c|c|c|c|c|}
\hline Variáveis & $p$-value & $\begin{array}{l}\text { Odds ratio bruto } \\
\text { (intervalo de } \\
\text { confiança 95\%) }\end{array}$ & $p$-value & $\begin{array}{l}\text { Odds ratio ajustado } \\
\text { (intervalo de } \\
\text { confiança 95\%) }\end{array}$ \\
\hline \multicolumn{5}{|l|}{$\begin{array}{l}\text { Exaustão } \\
\text { Emocional }\end{array}$} \\
\hline \multicolumn{5}{|l|}{ Workaholism } \\
\hline $\begin{array}{l}\text { Trabalho } \\
\text { compulsivo }\end{array}$ & $<0,001$ & $6,109(3,890-9,593)$ & $<0,001$ & $6,440(3,951-10,496)$ \\
\hline $\begin{array}{l}\text { Trabalho } \\
\text { excessivo }\end{array}$ & $<0,001$ & $4,008(2,592-6,197)$ & $<0,001$ & $3,579(2,257-5,673)$ \\
\hline Workaholic & $<0,001$ & $7,874(4,741-13,075)$ & $<0,001$ & $7,548(4,425-12,876)$ \\
\hline $\begin{array}{l}\text { Trabalhador } \\
\text { positivo }\end{array}$ & $<0,001$ & $0,232(0,148-0,364)$ & $<0,001$ & $0,236(0,145-0,384)$ \\
\hline \multicolumn{5}{|c|}{ Qualidade de vida } \\
\hline Overall & $<0,001$ & $0,103(0,047-0,223)$ & $<0,001$ & $0,113(0,051-0,250)$ \\
\hline Física & $<0,001$ & $0,083(0,050-0,137)$ & $<0,001$ & $0,091(0,054-0,154)$ \\
\hline Psicológica & $<0,001$ & $0,124(0,076-0,201)$ & $<0,001$ & $0,139(0,083-0,230)$ \\
\hline Social & $<0,001$ & 0,258(0,167-0,398) & $<0,001$ & $0,310(0,197-0,487)$ \\
\hline Ambiental & $<0,001$ & $0,179(0,113-0,281)$ & $<0,001$ & $0,205(0,128-0,330)$ \\
\hline \multicolumn{5}{|c|}{ Despersonalização } \\
\hline \multicolumn{5}{|l|}{ Workaholism } \\
\hline $\begin{array}{l}\text { Trabalho } \\
\text { compulsivo }\end{array}$ & $<0,001$ & $2,918(1,909-4,460)$ & $<0,001$ & $2,831(1,792-4,471)$ \\
\hline $\begin{array}{l}\text { Trabalho } \\
\text { excessivo }\end{array}$ & $<0,001$ & $3,394(2,209-5,214)$ & $<0,001$ & $2,884(1,827-4,553)$ \\
\hline Workaholic & $<0,001$ & 4,337(2,737-6,874) & $<0,001$ & $3,935(2,410-6,424)$ \\
\hline $\begin{array}{l}\text { Trabalhador } \\
\text { positivo }\end{array}$ & $<0,001$ & $0,362(0,234-0,560)$ & $<0,001$ & $0,398(0,248-0,639)$ \\
\hline \multicolumn{5}{|c|}{ Qualidade de vida } \\
\hline Overall & $<0,001$ & $0,304(0,165-0,558)$ & $<0,001$ & $0,312(0,165-0,591)$ \\
\hline Física & $<0,001$ & 0,302(0,197-0,464) & $<0,001$ & $0,353(0,221-0,561)$ \\
\hline Psicológica & $<0,001$ & $0,232(0,148-0,362)$ & $<0,001$ & $0,247(0,152-0,401)$ \\
\hline Social & $<0,001$ & 0,281(0,183-0,433) & $<0,001$ & $0,336(0,213-0,530)$ \\
\hline Ambiental & $<0,001$ & $0,330(0,214-0,508)$ & $<0,001$ & $0,421(0,266-0,665)$ \\
\hline \multicolumn{5}{|l|}{$\begin{array}{l}\text { Eficácia } \\
\text { Profissional }\end{array}$} \\
\hline \multicolumn{5}{|l|}{ Workaholism } \\
\hline $\begin{array}{l}\text { Trabalho } \\
\text { compulsivo }\end{array}$ & $<0,001$ & $2,478(1,629-3,770)$ & $<0,001$ & $2,416(1,530-3,817)$ \\
\hline $\begin{array}{l}\text { Trabalho } \\
\text { excessivo }\end{array}$ & $<0,001$ & $2,460(1,614-3,750)$ & 0,003 & $2,005(1,274-3,158)$ \\
\hline Workaholic & $<0,001$ & $3,320(2,105-5,237)$ & $<0,001$ & $2,934(1,801-4,780)$ \\
\hline $\begin{array}{l}\text { Trabalhador } \\
\text { positivo }\end{array}$ & $<0,001$ & $0,466(0,305-0,714)$ & 0,007 & $0,525(0,330-0,837)$ \\
\hline \multicolumn{5}{|c|}{ Qualidade de vida } \\
\hline Overall & $<0,001$ & $0,111(0,053-0,233)$ & $<0,001$ & $0,115(0,053-0,247)$ \\
\hline Física & $<0,001$ & $0,231(0,148-0,359)$ & $<0,001$ & $0,271(0,168-0,436)$ \\
\hline Psicológica & $<0,001$ & $0,205(0,130-0,324)$ & $<0,001$ & $0,227(0,139-0,373)$ \\
\hline Social & $<0,001$ & $0,217(0,140-0,337)$ & $<0,001$ & $0,250(0,156-0,398)$ \\
\hline Ambiental & $<0,001$ & $0,295(0,192-0,455)$ & $<0,001$ & $0,375(0,237-0,593)$ \\
\hline
\end{tabular}

As chances de alta exaustão emocional, alta despersonalização e baixa eficácia profissional foram 555,5\%, 249,1\% e 135,1\%, respectivamente, maiores entre os docentes com workaholism. Por outro lado, foram $85,7 \%, 59,2 \%$ e $86,5 \%$, respectivamente, menores nos docentes que consideraram ter uma alta qualidade de vida. 
Tabela 2. Modelos múltiplos de associação do burnout com 0 workaholism e qualidade de vida na amostra em estudo

\begin{tabular}{cccccc}
\hline Modelos Múltiplos & $p$-value & $\begin{array}{c}\text { Odds ratiobruto } \\
\text { (intervalo de } \\
\text { confiança 95\%) }\end{array}$ & p-value & $\begin{array}{c}\text { Odds ratio } \\
\text { (intervalo de } \\
\text { confiança 95\%) }\end{array}$ \\
\hline $\begin{array}{c}\text { Exaustão Emocional } \\
\text { Workaholism } \\
\text { Qualidade de } \\
\text { vida }\end{array}$ & $<0,001$ & $6,783(3,977-11,415)$ & $<0,001$ & $6,555(3,770-11,398)$ \\
$\begin{array}{c}\text { Despersonalização } \\
\text { Workaholism }\end{array}$ & $<0,001$ & $0,134(0,059-0,302)$ & $<0,001$ & $0,143(0,062-0,332)$ \\
$\begin{array}{c}\text { Qualidade de } \\
\text { vida }\end{array}$ & 0,006 & $0,415(0,221-0,781)$ & 0,008 & $0,408(0,210-0,792)$ \\
\hline $\begin{array}{c}\text { Eficácia Profissional } \\
\text { Workaholism }\end{array}$ & $<0,001$ & $2,645(1,644-4,256)$ & $<0,001$ & $2,351(1,411-3,920)$ \\
$\begin{array}{c}\text { Qualidade de } \\
\text { vida }\end{array}$ & $<0,001$ & $0,137(0,064-0,290)$ & $<0,001$ & $0,135(0,062-0,296)$ \\
\hline
\end{tabular}

Nagelkerke R Square: 0,370, 0,241, 0,295; Hosmer \& Lemeshow: 0,612, 0,698, 0,191; ajustado por sexo, idade, regime de trabalho, anos de docência no mestrado/doutorado, número de PPG de vínculo, receber bolsa produtividade, número de orientados no mestrado/doutorado e depressão

\section{Discussão}

Os resultados das análises múltiplas confirmaram amplamente o modelo hipotético de que os docentes workaholics e os que consideraram possuir baixa qualidade de vida em todos os seus aspectos estão esgotados em seus recursos mentais de energia. Esse dado está embasado na alegação de que o workaholism pode ser uma das causas bases do burnout, ${ }^{(17)}$ assim como, a baixa qualidade de vida. ${ }^{(7)}$ Em contrapartida, o trabalho positivo foi fator de proteção para o burnout. Outro estudo também identificou que comparado aos workaholics, os trabalhadores positivos são menos propensos a experimentar exaustão severa e cinismo. ${ }^{(3)}$

Demonstrou-se que cerca de um em cada três professores de mestrado e/ou doutorado têm indicativo de burnout e a mesma proporçáo ocorre para o workaholism. Esses são dados alarmantes se considerarmos o fato de que esses profissionais oferecem serviços altamente especializados e específicos, bem como pela contribuição social do seu trabalho. ${ }^{(14)}$ Garantir o bem-estar do professor no trabalho é essencial para garantir um ensino de alta qualidade, ${ }^{(18)}$ o que pode estender-se a produçáo de pesquisas de qualidade.

As cargas de trabalho na docência no ensino superior, sobretudo, em nível de mestrado/doutorado são permeadas pelas múltiplas atividades somadas aos curtos prazos para sua execução. ${ }^{(19)}$ Isso faz com que os professores trabalhem arduamente, sem considerar como sentem-se a respeito, nem quais são as razões subjacentes, o elemento central do trabalho excessivo. ${ }^{(3)}$ Pela exigência constante de produtividade acadêmica e pela competitividade, ${ }^{(20)}$ os docentes pensam frequente e persistentemente sobre o trabalho, mesmo quando não estão nele, caracterizando o trabalho compulsivo. ${ }^{(3)}$ Em ambas situaçôes, torna-os exaustos emocionalmente pelo tempo insuficiente para recuperação. ${ }^{(1,21)}$ Ainda, a intensificação laboral, o produtivismo acadêmico e a precarização do trabalho têm contribuído para o adoecimento dos docentes de universidades públicas brasileiras, considerando suas atividades simultâneas na graduação, pós-graduação e gestão. ${ }^{(2)}$

Quanto a relação entre baixa eficácia profissional e trabalho compulsivo e excessivo, diante da necessidade de dominar teorias e métodos e da dinamicidade da área da saúde, os docentes podem apresentar sentimentos de incompetência no trabalho e compensam trabalhando duro para se sentirem mais competentes, sendo esse processo permeado pelo perfeccionismo socialmente prescrito, autodisciplina, energia e ambição. ${ }^{(23-25)}$

O contexto socioeconômico e tecnológico impacta e redefine constantemente as características do trabalho docente, exigindo a sua reinvenção para atender as necessidades e tendências de ensino e produção de conhecimento, muitas vezes, desconsiderando sua subjetividade e autonomia. ${ }^{(26)}$ Além disso, o próprio uso das tecnologias de informação e comunicação aumenta a tendência ao workaholism e a carga de trabalho, pois torna tênue a linha entre vida dentro e fora do trabalho, ${ }^{(27)}$ sobretudo com o uso das redes sociais.

Desse modo, os docentes com workaholism gradualmente perdem seu entusiasmo para trabalhar, tratam com indiferença seus colegas de trabalho e alunos e, por consequência, perdem seu senso de realização. ${ }^{(28)}$ Os workaholics usam a concentração nas atividades laborais para evitar a participação em funçôes sociais no trabalho e como resultado eles têm má qualidade nos relacionamentos interpessoais e na percepção da qualidade de suas vidas. ${ }^{(29)}$

Esta pesquisa demonstrou que o workaholism é prejudicial à saúde biopsicossocial dos docentes pela magnitude de associação com o burnout, pois quando o trabalho torna-se a prioridade da vida, a saúde pode ser afetada, ${ }^{(28)}$ considerando que as pessoas com 
comportamento obsessivo-compulsivo são mais propensas ao adoecimento e a desregulação fisiológica. ${ }^{(30)}$ Tais afirmaçóes são corroboradas pela associação do burnout com a baixa percepção de qualidade de vida em todas as suas dimensóes. Outros estudos indicaram que o burnout esteve associado à uma qualidade de vida acentuadamente baixa, que estava afetando todas as áreas da vida, ${ }^{(31,32)}$ o que enfatiza a gravidade da condição e a necessidade de prevenção e detecção precoce.

A intensificaçáo do trabalho docente, o repouso insuficiente e o estado de exaustáo física contribuem para que os docentes sintam-se cansados e sem energia. ${ }^{(22)}$ Assim, tendem a não aproveitar a vida fora do trabalho, não percebem um significado em suas vidas, estão insatisfeitos consigo mesmos, com o sono, aparência física, concentração, capacidade de realizar atividades diárias e capacidade laboral. ${ }^{(32)}$ O esgotamento também associa-se aos sentimentos negativos, como mau humor, desespero, ansiedade e depressão. ${ }^{(33)}$ A dedicação exagerada à atividade docente modifica as relaçóes com a família e os amigos, o tempo de lazer e a vida cotidiana. ${ }^{(24,32)}$

Sobre as relaçôes sociais, tem-se demonstrado sua importância na manifestação da síndrome. A incivilidade laboral, expressa por comportamentos descorteses, condescendentes e desrespeitosos, na maioria das vezes não verbais, tem efeito mais forte sobre o burnout, sendo mais prejudicial para os indivíduos que estão mais comprometidos com suas organizaçôes. ${ }^{(34)}$ Em contraste, o apoio social recebido das pessoas do trabalho é essencial para evitar o burnout, devido a promoção de um clima amistoso e de apoio recíproco, inclusive entre docentes. ${ }^{(35)}$

Diante das múltiplas consequências individuais, organizacionais e sociais do burnout, torna-se premente que os gestores das instituiçóes de ensino superior e os próprios trabalhadores conscientizem-se do problema e empenhem-se para promover ambientes de trabalho mais saudáveis.

A limitação mais importante é a natureza transversal deste estudo que impede a determinação das relaçôes de causa e efeito. Todas as medidas utilizadas foram autorrelatos, em que as respostas podem ser influenciadas por valores pessoais e sociais, além disso há uma tendência de autonegação envolvida no burnout e no workaholism. ${ }^{(29)}$
No entanto, um ponto forte deste estudo é o recrutamento de docentes de todas as universidades públicas com PPG em Enfermagem no Brasil. Ainda, este estudo traz contribuições importantes, mostrando que o burnout está associado positiva e significativamente ao workaholism e a baixa qualidade de vida do professor de ensino superior que dedica-se ao ensino na pós-graduação.

\section{Conclusão}

As dimensóes do burnout foram estatisticamente associadas aos docentes com workaholism e que consideram ter baixa qualidade de vida. Várias medidas são necessárias para evitar o burnout do professor a serem implantadas nas instituiçóes, mas este estudo demonstra a importância da redução da carga laboral e a pressão de tempo na profissão. Torna-se relevante desenvolver uma cultura positiva de relacionamentos sociais na universidade, bem como elaborar programas de gerenciamento de estresse, de promoção da saúde e qualidade de vida que favorecerão um ambiente saudável de ensino.

\section{Agradecimentos}

Esta pesquisa foi parcialmente financiada pela Fundação Araucária por meio da Chamada Pública 09/2016 - Programa Institucional de Pesquisa Básica e Aplicada e pela Universidade Estadual do Norte do Paraná - UENP/PROPG/EDITORA UENP.

\section{Colaborações}

Galdino MJQ e Haddad MCFL contribuíram com a concepção do projeto, análise e interpretação dos dados, redação do artigo, revisão crítica do conteúdo intelectual e aprovação final da versão a ser publicada. Martins JT, Robazzi MLCC, Pelloso SM e Barreto MFC contribuíram na interpretação dos dados, revisão crítica relevante do conteúdo intelectual e aprovação final da versão a ser publicada. 


\section{Referências}

1. Skaalvik EM, Skaalvik S. Dimensions of teacher burnout: relations with potential stressors at school. Soc Psychol Educ. 2017;20(4):775-90.

2. Shojaei F, Shirazi M. Investigating the relationship between workaholism and occupational stress and life quality among female teachers at elementary schools (a case study of Torbat-e Jam). Int J Hum Cult Stud. 2016;(Spe):373-81.

3. Schaufeli WS, Shimazu A, Taris TW. Being driven to work excessively hard. Cross-Cultural Res. 2009;43(4):320-48.

4. Peng J, Li D, Zhang Z, Tian Y, Miao D, Xiao W, et al. How can core self-evaluations influence job burnout? The key roles of organizational commitment and job satisfaction. J Health Psychol. 2016;21(1):50-9.

5. Fleck MP, Louzada S, Xavier M, Cachamovich E, Vieira G, Santos L, et al. Application of the Portuguese version of the abbreviated instrument of quality life WHOQOL-bref. Rev Saude Publica. 2000;34(2):178-83.

6. Moyer F, Aziz S, Wuensch K. From workaholism to burnout: psychological capital as a mediator. Int J Workplace Health Manag. 2017;10(3):213-27.

7. Grigorescu S, Cazan AM, Grigorescu OD, Rogozea LM. The role of the personality traits and work characteristics in the prediction of the burnout syndrome among nurses-a new approach within predictive, preventive, and personalized medicine concept. EPMA J. 2018;9(4):355-65.

8. Kwak Y, Kim JS, Han Y, Seo Y. The effect of work addiction on Korean nurses' professional quality of life: a cross-sectional study. J Addict Nurs. 2018;29(2):119-27.

9. Nonnis M, Massidda D, Cuccu S, Cortese CG. The impact of workaholism on nurses' burnout and disillusion. Open Psychol J. 2018;11(1):77-88.

10. Leiter MP, Maslach C. Latent burnout profiles: a new approach to understanding the burnout experience. Burn Res. 2016;3(4):89-100.

11. Maslach C, Leiter MP. Understanding the burnout experience: recent research and its implications for psychiatry. World Psychiatry. 2016;15(2):103-11.

12. Meredith C, Schaufeli W, Struyve C, Vandecandelaere M, Gielen S, Kyndt E. Burnout contagion' among teachers: A social network approach. J Occup Organ Psychol. 2019. https://doi.org/10.1111/joop.12296.

13. Salvagioni DA, Melanda FN, Mesas AE, González AD, Gabani FL, Andrade SM. Physical, psychological and occupational consequences of job burnout: A systematic review of prospective studies. PLoS One. 2017;12(10):e0185781.

14. Bøgelund P. How supervisors perceive PhD supervision: and how they practice it'. Int J Dr Stud. 2015;10:39-55.

15. Carlotto MS, Câmara SC. Factorial analysis of the Maslach Burnout Inventory $(\mathrm{MBI})$ in a sample of teachers from private schools. Psicol Estud. 2004;9(3):499-505.

16. Carlotto MS, Miralles MD. Translation, adaptation and exploration of psychometric properties of "Dutch Work Addiction Scale" (DUWAS). Contextos Clín. 2010;3(2):141-50.

17. Gonçalves G, Brito F, Sousa S, Santos J. Workaholism and burnout: antecedents and effects. In: Arezes PM, Baptista JS, Barroso MP, Carneiro P, Cordeiro P, Costa N, et al., editors. Occupational Safety and Hygiene V. London: Taylor \& Francis Group; 2017. p. 53-7.

18. Zee M, Koomen HM. Teacher self-efficacy and its effects on classroom processes, student academic adjustment, and teacher well-being a synthesis of 40 years of research. Rev Educ Res. 2016;86(4):9811015.

19. Souto BL, Beck CL, Trindade LR, Silva RM, Backes DS, Bastos RM. The teaching work in the post-graduation program: pleasure and suffering. Rev Enferm UFSM. 2017;7(1):29-39.

20. Smeltzer SC, Cantrell MA, Sharts-Hopko NC, Heverly MA, Jenkinson A, Nthenge S. Assessment of the impact of teaching demands on research productivity among doctoral nursing program faculty. J Prof Nurs. 2016;32(3):180-92.

21. Sezgin Nartgun Ş, Ekinci S, Tukel H, Limon I.Teacher views regarding workaholism and occupational professionalism. Univers J Educ Res. 2016;4 12A:112-8.

22. Souza KR, Mendonça AL, Rodrigues AM, Felix EG, Teixeira LR, Santos $\mathrm{MB}$, et al. [The new organization of labor at public universities: collective consequences of job instability on the health of teachers]. Cien Saude Colet. 2017;22(11):3667-76.

23. Andreassen CS, Bjorvatn B, Moen BE, Waage S, Magerøy N, Pallesen $S$. A longitudinal study of the relationship between the five-factor model of personality and workaholism. TPM Test Psychom Methodol Appl Psychol. 2016;23(3):285-98.

24. Clark MA, Michel JS, Zhdanova L, Shuang Y, Pui SY, Baltes BB. All work and no play? A meta-analytic examination of the correlates and outcomes of workaholism. J Manage. 2016;42(7):1836-73.

25. Gillet N, Morin AJ, Cougot B, Gagné M. Workaholism profiles: associations with determinants, correlates, and outcomes. J Occup Organ Psychol. 2017;90(4):559-86.

26. Ruza FM, Silva EP. The productive transformations in the postgraduate: the pleasure in the teaching work suspended? Rev Subj. 2016;16(1):91-103.

27. Molino M, Cortese CG, Ghislieri C. Unsustainable working conditions: the association of destructive leadership, use of technology, and workload with workaholism and exhaustion. Sustainability. 2019;11(2):446.

28. Nie Y, Sun H. Why do workaholics experience depression? A study with Chinese University teachers. J Health Psychol. 2016;21(10):2339-46.

29. Hamidizadeh $A$, Koolivand $H$, Hajkarimi F. Is workaholism antecedent of burn out? Eur J Acad Essays. 2014;1(8):1-9.

30. Salanova M, López-González AA, Llorens S, Del Líbano M, VicenteHerrero MT, Matias Tomás-Salvá M. Your work may be killing you! Workaholism, sleep problems and cardiovascular risk. Work Stress. 2016;30(3):228-42.

31. Grensman A, Acharya BD, Wändell P, Nilsson G, Werner S. Healthrelated quality of life in patients with Burnout on sick leave: descriptive and comparative results from a clinical study. Int Arch Occup Environ Health. 2016;89(2):319-29.

32. Alves PC, Oliveira AF, Paro HB. Quality of life and burnout among faculty members: how much does the field of knowledge matter? PLoS One. 2019;14(3):e0214217.

33. Yao SM, Yu HM, Ai YM, Song PP, Meng SY, Li W. Job-related burnout and the relationship to quality of life among Chinese medical college staff. Arch Environ Occup Health. 2015;70(1):27-34.

34. Liu W, Zhou ZE, Che XX. Effect of workplace incivility on OCB through burnout: the moderating role of affective commitment. J Bus Psychol. 2019;(5):657-69.

35. Ju C, Lan J, Li Y, Feng W, You X. The mediating role of workplace social support on the relationship between trait emotional intelligence and teacher burnout. Teach Teach Educ. 2015;51:58-67. 